\title{
Bemerkung zu der Abhandlung: Über einige mit dem Satz von Kakeya verwandte Sätze.
}

(Diese Zeitschrift 37 (1933), S. 61-76.)

\section{Von}

L. Berwald in Prag.

Herr St. Lipka hat mich freundlichst darauf aufmerksam gemacht, daB sich von Hilfssatz 5 meiner Arbeit der besondere Fall $r=0$, auf dem allein mein Beweis des dortigen Satzes 1 beruht, schon in seiner Abhandlung: Eine Verallgemeinerung des Rouchéschen Satzes (Journ. f. d. reine u. angew. Math. 160 (1929), S. 143-150) auf S. 143 findet.

\section{Bemerkung zu der Arbeit: Über das Dirichletsche Problem im Großen für nichtlineare elliptische Differentialgleichungen.}

(Diese Zeitschrift 37 (1933), S. 623-634.)

$$
\text { Von }
$$

J. Schauder in Lemberg.

Wie in einer gemeinsamen Arbeit von Herrn J. Leray und von mir gezeigt wird, kann mein in der Einleitung ausgesprochener Satz wesentlich verallgemeinert werden. Man kann nämlich alle Eindeutigkeitsvoraussetzungen fallen lassen, und dennoch bleibt die sich auf Gleichung (3) beziehende Behauptung von der Existenz der Lösung bestehen. Die Überlegungen der erwähnten Arbeit sind aber für den Beweis des erweiterten Satzes notwendig. Ähnliches gilt für allgemeine elliptische Differentialgleichungen. 